\title{
Impact of prevention in a tuberculosis model with latent delay
}

Jun Li* and Mingju Ma

\section{*Correspondence:}

lijun@xidian.edu.cn

School of Mathematics and

Statistics, Xidian University, Xi'an,

710071, P.R. China

\section{囪 Springer}

\begin{abstract}
An tuberculosis model with prevention effect and latent delay is investigated. To the best of our knowledge, it is the first work to introduce a nonlinear incidence rate to describe the prevention effect. The sharp threshold condition $R_{0}$ of our model is obtained. Meanwhile, we also derive the globally dynamics of system and illustrate the analytical results by numerical simulations. Finally, the influence of the prevention effect is discussed.
\end{abstract}

MSC: $92 \mathrm{D} 25 ; 34 \mathrm{C} 25$

Keywords: disease ecology; delay; globally asymptotic stability

\section{Introduction}

Tuberculosis (TB) is a chronic disease caused by mycobacterium tuberculosis infection. According to the world health organization report, a third of the people in the world carry mycobacterium tuberculosis. Every year, there are 8 million people to become new infection cases of TB, $95 \%$ of which are in developing countries and whose ages range from 15-59. There are 4.5 million active-tuberculosis patients and 1.5 million infectious ones in China. The TB death toll is 130,000 each year, which is far more than the total of the other infectious epidemic diseases [1]. The disease spreads through the air. The mycobacterium tuberculosis is released into the air when TB patients cough, sneeze, talk, or spit. Only a small amount of bacteria inhaled can cause infection, thus it has strong effects and is not easy to efficiently prevent. Some people infected with TB bacilli will get the disease, immediately, and the others become TB patients after a period of incubation. The incubation period may be short, but it may also be long; even for decades. In recent years, the TB worldwide has been controlled effectively for the invention and wide application of antibiotics. But the mycobacterium tuberculosis develops resistance to the existing antibiotics more or less because of the excessive abuse of antibiotics, so it is still essential for the prevention and control of tuberculosis.

Mathematical models have effects on the effective prediction for infectious disease prevention and control. However, the incidence rates are bilinear or standard in most of models. These kinds of rates are accurate in the early time of the outbreak of infectious diseases or the low quantity of population, and the dynamics properties are relatively simple. For example, Huo and Dang [2] studied a TB model with bilinear incidence rate and different incubations. They obtained two epidemic threshold values, which could be used as

(c) $2016 \mathrm{Li}$ and Ma. This article is distributed under the terms of the Creative Commons Attribution 4.0 International License (http://creativecommons.org/licenses/by/4.0/), which permits unrestricted use, distribution, and reproduction in any medium, provided you give appropriate credit to the original author(s) and the source, provide a link to the Creative Commons license, and indicate if changes were made. 
the prevalent conditions of long and short incubations. In addition, the standard incidence rate is applied in [3]. In this paper, the author studied a TB model with isolation and incomplete treatment. They prove the global stability of endemic equilibrium by the generalized Dulac-Bendixson criterion and obtain the basic reproductive number of TB outbreak. Other different TB models have been studied in [4-7] and references therein.

The bilinear and standard incidence rates are more simple to analyse in mathematics, however, the nonlinear incidence rate is more precise in describing the effect of special groups $[8,9]$. In this paper, we introduce a nonlinear incidence rate to reflect the change of the psychological effect of people due to understanding for tuberculosis knowledge. Generally, if someone knows the high pathogenicity of TB bacteria, he will be on guard consciously in contacting with TB patients. Thus, the factor is considered of the incidence rate, and we use $\beta I /(1+a S+b I+c E+d R)$ representing the effective contact rate between susceptible and infected individuals. Here, $a, b, c, d(\geq 0)$ denote the level of prevention of susceptible individuals, infected individuals, lurkers, and recovered population, respectively. The higher the value is, the stronger the prevention ability becomes. There is no precaution if the value is zero. For simplicity, we make the assumption of $c=d=0$ in the paper. The total number of susceptible individuals entering the incubation $\beta S I /(1+a S+b I)$ in unit time, which represents the Beddington-DeAngelis (B-D) functional response function in general predator-prey models, has different meanings in our phthisis model. On the other hand, as described in [3], once infected, phthisis is extremely difficult to cure thoroughly. Therefore, we consider that part of recovered individuals will still fall back to the disease. At the same time, we also add the latent delay factor. The model is as follows:

$$
\begin{aligned}
& \frac{d S(t)}{d t}=A-\mu S(t)-\frac{\beta S(t) I(t)}{1+a S(t)+b I(t)}, \\
& \frac{d E(t)}{d t}=\frac{\beta S(t) I(t)}{1+a S(t)+b I(t)}-\mu E(t)-\frac{\beta e^{-\mu \tau} S(t-\tau) I(t-\tau)}{1+a S(t-\tau)+b I(t-\tau)}, \\
& \frac{d I(t)}{d t}=\frac{\beta e^{-\mu \tau} S(t-\tau) I(t-\tau)}{1+a S(t-\tau)+b I(t-\tau)}+\delta R(t)-(\mu+\gamma+\epsilon) I(t), \\
& \frac{d R(t)}{d t}=\gamma I(t)-(\mu+\delta) R(t),
\end{aligned}
$$

where $S(t), E(t), I(t)$ and $R(t)$ are the number of susceptible, exposed, infected, and recovered individuals. $A$ is the recruitment (or birth) rate, $\mu$ is the natural death rate, $\beta$ is the transmission coefficient, $\tau$ is the time delay which represents the latent period of the disease, $\delta$ represents the rate that a recovered individual reverts into the infected compartment, $\gamma$ is the rate at which infective individuals recover, and $\epsilon$ is the death rate induced by the disease. We take the initial conditions

$$
S(\theta)=\phi_{1}(\theta), \quad I(\theta)=\phi_{2}(\theta), \quad \phi_{i}(\theta) \geq 0, \theta \in[-\tau, 0], \phi_{i}(0)>0, i=1,2,
$$

where $\left(\phi_{1}(\theta), \phi_{2}(\theta)\right) \in C\left([-\tau, 0], R_{+0}^{2}\right)$ and $R_{+0}^{2}=\left\{\left(x_{1}, x_{2}\right): x_{i} \geq 0, i=1,2\right\}$.

As regards the continuity of the initial conditions, we also assume

$$
E(0)=\int_{-\tau}^{0} \beta e^{\mu \tau} \frac{\phi_{1}(\theta) \phi_{2}(\theta)}{1+a \phi_{1}(\theta)(\theta)+b \phi_{2}(\theta)} d \theta .
$$


From [10], the unique solution of system (1.1) satisfying the initial conditions (1.2) and (1.3) can be obtained. We also can easily show that all the solutions of (1.1) defined on $[0,+\infty)$ will remain positive for all $t \geq 0$.

In the following sections, we will establish the global dynamics of system (1.1). The organization of this paper is as follows. In Section 2, the local stability of disease-free equilibrium and epidemic equilibrium is given. We will show the global dynamics in Section 3. At last, a brief discussion is presented in Section 4.

\section{Local stability}

Denote $R_{0}=\frac{A \beta e^{-\mu \tau}(\mu+\delta)}{(a A+\mu)(\delta(\epsilon+\mu)+\mu(\mu+\gamma+\epsilon))}$. Next, we will show $R_{0}$ is the threshold condition of the epidemic outbreaks. The model always has a disease-free equilibrium $E_{1}(A / \mu, 0,0,0)$. But when $R_{0}>1$, there also is an epidemic equilibrium $E_{2}\left(S^{*}, E^{*}, I^{*}, R^{*}\right)$ satisfying

$$
\begin{aligned}
S^{*} & =\frac{\alpha\left(1+b I^{*}\right)}{\beta e^{-\mu \tau}-a \alpha}, \quad E^{*}=\frac{\alpha\left(e^{\mu \tau}-1\right)}{\mu} I^{*}, \quad R^{*}=\frac{\gamma}{\mu+\delta} I^{*}, \\
I^{*} & =\frac{A\left(\beta e^{-\mu \tau}-a \alpha\right)-\mu \alpha}{\mu \alpha b+\beta \alpha-a \alpha^{2} e^{\mu \tau}} \\
& =\frac{(a A+\mu)(\mu+\delta)}{\beta(\mu+\delta)+b \mu(\mu+\delta)-a e^{\mu \tau}(\delta(\mu+\epsilon)+\mu(\mu+\gamma+\epsilon))}\left(R_{0}-1\right),
\end{aligned}
$$

where $\alpha=\mu+\gamma+\epsilon-\frac{\gamma \delta}{\mu+\delta}$. In fact, since $R_{0}>1$,

$$
\begin{aligned}
A \beta e^{-\mu \tau}(\mu+\delta) & >(a A+\mu)(\delta(\epsilon+\mu)+\mu(\mu+\gamma+\epsilon)) \\
& >a A(\delta(\epsilon+\mu)+\mu(\mu+\gamma+\epsilon))
\end{aligned}
$$

which means

$$
\beta(\mu+\delta)>a e^{\mu \tau}(\delta(\epsilon+\mu)+\mu(\mu+\gamma+\epsilon))
$$

so, if $R_{0}>1, E_{2}\left(S^{*}, E^{*}, I^{*}, R^{*}\right)$ always exists. The characteristic equation of system (1.1) at the disease-free equilibrium has the form of

$$
(\lambda+\mu)^{2}\left(\lambda^{2}+P_{1}(\tau) \lambda+P_{0}(\tau)+\left(Q_{1}(\tau) \lambda+Q_{0}(\tau)\right) e^{-\lambda \tau}\right)=0
$$

where

$$
\begin{aligned}
& P_{1}(\tau)=\mu+\delta+\mu+\gamma+\epsilon, \quad P_{0}(\tau)=(\mu+\delta)(\mu+\gamma+\epsilon)-\delta \gamma, \\
& Q_{1}(\tau)=-\frac{\beta A e^{-\mu \tau}}{\mu+a A}, \quad Q_{0}(\tau)=-(\mu+\delta) \frac{\beta A e^{-\mu \tau}}{\mu+a A} .
\end{aligned}
$$

Obviously, equation (2.2) has a negative real root $\lambda=-\mu$, and the others can be determined by

$$
\lambda^{2}+P_{1}(\tau) \lambda+P_{0}(\tau)+\left(Q_{1}(\tau) \lambda+Q_{0}(\tau)\right) e^{-\lambda \tau}=0
$$

When $\tau=0,(2.3)$ becomes

$$
\lambda^{2}+\left(P_{1}(0)+Q_{1}(0)\right) \lambda+\left(P_{0}(0)+Q_{0}(0)\right)=0 .
$$


By calculation,

$$
\begin{aligned}
& P_{1}(0)+Q_{1}(0)=\frac{(\mu+\gamma+\epsilon) \mu+\delta(\mu+\epsilon)}{\mu+\delta}\left(1-R_{0}\right)+\frac{(\mu+\delta+\gamma)(\mu+\delta)-\mu \gamma}{\mu+\delta}>0, \\
& P_{0}(0)+Q_{0}(0)=((\mu+\gamma+\epsilon) \mu+\delta(\mu+\epsilon))\left(1-R_{0}\right)>0,
\end{aligned}
$$

hence, if $\tau=0, E_{1}$ is locally asymptotically stable.

When $\tau>0$, if $i \omega(\omega>0)$ is a solution of (2.3), from (2.3), we have

$$
\begin{aligned}
& \omega^{2}-P_{0}(\tau)=Q_{0}(\tau) \cos \omega \tau+Q_{1}(\tau) \omega \sin \omega \tau, \\
& P_{1}(\tau) \omega=Q_{0}(\tau) \sin \omega \tau-Q_{1}(\tau) \omega \cos \omega \tau,
\end{aligned}
$$

squaring and adding the equations above, we have

$$
\omega^{4}+\left(P_{1}^{2}(\tau)-2 P_{0}(\tau)-Q_{1}^{2}(\tau)\right) \omega^{2}+P_{0}^{2}(\tau)-Q_{0}^{2}(\tau)=0 .
$$

Obviously, $P_{0}^{2}(\tau)-Q_{0}^{2}(\tau)>0$, on the other hand,

$$
\begin{aligned}
& P_{1}^{2}(\tau)-2 P_{0}(\tau)-Q_{1}^{2}(\tau) \\
& \quad=\frac{1}{(\mu+\delta)^{2}}\left((\mu+\delta)^{4}+\delta^{2}\left(\gamma(2 \mu+2 \epsilon+\gamma)+2 \delta \gamma(\mu+\delta)^{2}+2 \mu \delta \gamma(\mu+\gamma+\epsilon)\right)\right) \\
& \quad>0 .
\end{aligned}
$$

Hence, if $R_{0}<1$, equation (2.4) has no real root. Notice that the disease-free equilibrium $E_{1}$ is locally asymptotic stable when $\tau=0$. From Theorem 4.1 in [11], we know that $E_{1}$ is local asymptotic stable if $R_{0}<1$. Denote

$$
f(\lambda)=\lambda^{2}+P_{1}(\tau) \lambda+P_{0}(\tau)+\left(Q_{1}(\tau) \lambda+Q_{0}(\tau)\right) e^{-\lambda \tau} .
$$

If $R_{0}>1$,

$$
f(0)=\left(1-R_{0}\right)(\mu(\mu+\gamma+\epsilon)+\delta(\mu+\epsilon))<0 .
$$

On the other hand, $\lim _{\lambda \rightarrow+\infty} f(\lambda)=+\infty$, equation (2.2) has one positive real root at least, so if $R_{0}>1, E_{1}$ is unstable. Next, we will show the stability of epidemic equilibrium $E_{2}$. The characteristic equation of system (1.1) at $E_{2}$ is

$$
(\lambda+\mu)\left(\lambda^{3}+p_{2}(\tau) \lambda^{2}+p_{1}(\tau) \lambda+p_{0}+\left(q_{2}(\tau) \lambda^{2}+q_{1}(\tau) \lambda+q_{0}\right) e^{-\lambda \tau}\right)=0,
$$

where

$$
\begin{aligned}
& p_{2}(\tau)=3 \mu+\delta+\gamma+\epsilon+\frac{\beta I^{*}\left(1+b I^{*}\right)}{\left(1+a S^{*}+b I^{*}\right)^{2}}, \\
& p_{1}(\tau)=\left(\mu+\frac{\beta I^{*}\left(1+b I^{*}\right)}{\left(1+a S^{*}+b I^{*}\right)^{2}}\right)(2 \mu+\delta+\gamma+\epsilon)+\alpha(\mu+\delta),
\end{aligned}
$$




$$
\begin{aligned}
& p_{0}(\tau)=\left(\mu+\frac{\beta I^{*}\left(1+b I^{*}\right)}{\left(1+a S^{*}+b I^{*}\right)^{2}}\right)(\mu+\delta) \alpha, \\
& q_{0}(\tau)=-\frac{\alpha \mu(\mu+\delta)\left(1+a S^{*}\right)}{1+a S^{*}+b I^{*}}, \\
& q_{1}(\tau)=-(2 \mu+\delta) \frac{\alpha\left(1+a S^{*}\right)}{1+a S^{*}+b I^{*}} \\
& q_{2}(\tau)=-\alpha \frac{1+a S^{*}}{1+a S^{*}+b I^{*}} .
\end{aligned}
$$

Equation (2.5) has a negative real $\operatorname{root} \lambda=-\mu$, and the other roots will be determined by

$$
\lambda^{3}+p_{2}(\tau) \lambda^{2}+p_{1}(\tau) \lambda+p_{0}(\tau)+\left(q_{2}(\tau) \lambda^{2}+q_{1}(\tau) \lambda+q_{0}(\tau)\right) e^{-\lambda \tau}=0
$$

If $\tau=0$, equation (2.7) becomes

$$
\lambda^{3}+\left(p_{2}(0)+q_{2}(0)\right) \lambda^{2}+\left(p_{1}(0)+q_{1}(0)\right) \lambda+\left(p_{0}(0)+q_{0}(0)\right)=0
$$

If $R_{0}>1$,

$$
\begin{aligned}
p_{2}(\tau)+q_{2}(\tau)= & 2 \mu+\delta+\frac{\delta \gamma\left(1+a S^{*}\right)}{(\mu+\delta)\left(1+a S^{*}+b I^{*}\right)}+\frac{b(\mu+\gamma+\epsilon) I^{*}}{\left(1+a S^{*}+b I^{*}\right)} \\
& +\frac{\beta I^{*}\left(1+b I^{*}\right)}{\left(1+a S^{*}+b I^{*}\right)^{3}}>0, \\
p_{1}(\tau)+q_{1}(\tau)= & (2 \mu+\delta+\gamma+\epsilon)\left(\mu+\frac{\beta I^{*}\left(1+b I^{*}\right)}{\left(1+a S^{*}+b I^{*}\right)^{2}}\right)-\frac{\mu \alpha\left(1+a S^{*}\right)}{1+a S^{*}+b I^{*}} \\
& +\frac{\alpha b(\mu+\delta) I^{*}}{1+a S^{*}+b I^{*}}>0, \\
p_{0}(\tau)+q_{0}(\tau)= & \alpha(\mu+\delta)\left(\mu+\frac{\beta I^{*}\left(1+b I^{*}\right)}{\left(1+a S^{*}+b I^{*}\right)^{2}}-\frac{\mu\left(1+a S^{*}\right)}{1+a S^{*}+b I^{*}}\right)>0,
\end{aligned}
$$

and

$$
\begin{aligned}
& \left(p_{1}(\tau)+q_{1}(\tau)\right)\left(p_{2}(\tau)+q_{2}(\tau)\right)-\left(p_{0}(\tau)+q_{0}(\tau)\right) \\
& =\left(\left(\mu+\frac{\beta I^{*}\left(1+b I^{*}\right)}{\left(1+a S^{*}+b I^{*}\right)^{2}}\right)(2 \mu+\delta+\gamma+\epsilon)-\frac{\mu \alpha\left(1+a S^{*}\right)}{1+a S^{*}+b I^{*}}+\frac{\alpha b(\mu+\delta) I^{*}}{1+a S^{*}+b I^{*}}\right) \\
& \quad \times\left(\frac{\delta \gamma\left(1+a S^{*}\right)}{(\mu+\delta)\left(1+a S^{*}+b I^{*}\right)}+\frac{I^{*}}{1+a S^{*}+b I^{*}}\left(b(\mu+\gamma+\epsilon)+\frac{\beta\left(1+b I^{*}\right)}{\left(1+a S^{*}+b I^{*}\right)^{2}}\right)\right) \\
& \quad+(2 \mu+\delta)\left(\mu(2 \mu+\delta+\gamma+\epsilon)-\frac{\mu \alpha\left(1+a S^{*}\right)}{1+a S^{*}+b I^{*}}\right) \\
& \quad+(2 \mu+\delta)\left\{(2 \mu+\delta+\gamma+\epsilon) \frac{\beta I^{*}\left(1+b I^{*}\right)}{\left(1+a S^{*}+b I^{*}\right)^{2}}+\frac{\alpha b(\mu+\delta) I^{*}}{1+a S^{*}+b I^{*}}\right. \\
& \left.\quad-\alpha(\mu+\delta)\left(\mu+\frac{\beta I^{*}\left(1+b I^{*}\right)}{\left(1+a S^{*}+b I^{*}\right)^{2}}-\frac{\mu\left(1+a S^{*}\right)}{1+a S^{*}+b I^{*}}\right)\right\} \\
& =F_{1}+F_{2}+F_{3},
\end{aligned}
$$


and it is easy to prove that

$$
\begin{aligned}
F_{1}= & \left\{\left(\mu+\frac{\beta I^{*}\left(1+b I^{*}\right)}{\left(1+a S^{*}+b I^{*}\right)^{2}}\right)(2 \mu+\delta+\gamma+\epsilon)-\frac{\mu \alpha\left(1+a S^{*}\right)}{1+a S^{*}+b I^{*}}+\frac{\alpha b(\mu+\delta) I^{*}}{1+a S^{*}+b I^{*}}\right\} \\
& \times\left\{\frac{\delta \gamma\left(1+a S^{*}\right)}{(\mu+\delta)\left(1+a S^{*}+b I^{*}\right)}+\frac{I^{*}}{1+a S^{*}+b I^{*}}\left(b(\mu+\gamma+\epsilon)+\frac{\beta\left(1+b I^{*}\right)}{\left(1+a S^{*}+b I^{*}\right)^{2}}\right)\right\}>0, \\
F_{2}= & (2 \mu+\delta)\left\{\mu(2 \mu+\delta+\gamma+\epsilon)-\frac{\mu \alpha\left(1+a S^{*}\right)}{1+a S^{*}+b I^{*}}\right\}>0, \\
F_{3}= & (2 \mu+\delta)\left\{(2 \mu+\delta+\gamma+\epsilon) \frac{\beta I^{*}\left(1+b I^{*}\right)}{\left(1+a S^{*}+b I^{*}\right)^{2}}+\frac{\alpha b(\mu+\delta) I^{*}}{1+a S^{*}+b I^{*}}\right. \\
& \left.-\alpha(\mu+\delta)\left(\mu+\frac{\beta I^{*}\left(1+b I^{*}\right)}{\left(1+a S^{*}+b I^{*}\right)^{2}}-\frac{\mu\left(1+a S^{*}\right)}{1+a S^{*}+b I^{*}}\right)\right\}>0 .
\end{aligned}
$$

Hence, if $R_{0}>1$, equation (2.7) has no real positive root. $E_{2}$ is local asymptotic stable when $\tau=0$.

When $\tau>0$, we denote by $i \omega(\omega>0)$ a root of equation (2.7), we have

$$
\begin{aligned}
& p_{1}(\tau) \omega-\omega^{3}=\left(q_{0}(\tau)-q_{2}(\tau) \omega^{2}\right) \sin \omega \tau-q_{1}(\tau) \omega \cos \omega \tau, \\
& p_{2}(\tau) \omega^{2}-p_{0}(\tau)=\left(q_{0}(\tau)-q_{2}(\tau) \omega^{2}\right) \cos \omega \tau+q_{1}(\tau) \omega \sin \omega \tau .
\end{aligned}
$$

Squaring and adding the two equations above, we have

$$
\omega^{6}+a_{4,0} \omega^{4}+a_{2,0} \omega^{2}+a_{0,0}=0
$$

where

$$
\begin{aligned}
a_{4,0}= & p_{2}^{2}(\tau)-2 p_{1}(\tau)-q_{2}^{2}(\tau) \\
= & \left(\mu+\frac{\beta I^{*}\left(1+b I^{*}\right)}{1+a S^{*}+b I^{*}}\right)^{2}+(\mu+\delta)^{2}+(\mu+\gamma+\epsilon)^{2}+2 \delta \gamma-\alpha^{2}>0, \\
a_{2,0}= & p_{1}^{2}(\tau)-2 p_{0}(\tau) p_{2}(\tau)+2 q_{0}(\tau) q_{2}(\tau)-q_{1}^{2}(\tau) \\
= & \left(\mu+\frac{\beta I^{*}\left(1+b I^{*}\right)}{\left(1+a S^{*}+b I^{*}\right)^{2}}\right)^{2}\left\{(\mu+\gamma+\epsilon)^{2}+(\mu+\delta)^{2}+2 \delta \gamma\right\}+\alpha^{2}(\mu+\delta)^{2} \\
& +\frac{\alpha^{2}\left(2 \mu(\mu+\delta)-(2 \mu+\delta)^{2}\right)\left(1+a S^{*}\right)^{2}}{\left(1+a S^{*}+b I^{*}\right)^{2}} \\
> & \left(\mu+\frac{\beta I^{*}\left(1+b I^{*}\right)}{\left(1+a S^{*}+b I^{*}\right)^{2}}\right)^{2}\left\{(\mu+\gamma+\epsilon)^{2}+(\mu+\delta)^{2}+2 \delta \gamma\right\} \\
& +\alpha^{2}(\mu+\delta)^{2}+\alpha^{2}\left(-2 \mu^{2}-2 \delta \mu-\delta^{2}\right) \\
= & \left(\mu+\frac{\beta I^{*}\left(1+b I^{*}\right)}{\left(1+a S^{*}+b I^{*}\right)^{2}}\right)^{2}\left\{(\mu+\gamma+\epsilon)^{2}+(\mu+\delta)^{2}+2 \delta \gamma\right\} \\
& -\mu^{2}\left(\mu+\gamma+\epsilon-\frac{\gamma \delta}{\mu+\delta}\right)^{2}>0, \\
a_{0,0}= & p_{0}^{2}(\tau)-q_{0}^{2}(\tau)=\alpha^{2}(\mu+\delta)^{2}\left\{\left(\mu+\frac{\beta I^{*}\left(1+b I^{*}\right)}{\left(1+a S^{*}+b I^{*}\right)^{2}}\right)^{2}-\frac{\mu^{2}\left(1+a S^{*}\right)^{2}}{\left(1+a S^{*}+b I^{*}\right)^{2}}\right\}>0 .
\end{aligned}
$$


Hence, if $R_{0}>1$, equation (2.7) has no positive roots. $E_{2}$ is locally asymptotically stable when $\tau=0$; from [11] Theorem 3.4.1, we see that if $R_{0}>1, E_{2}$ exists and is locally asymptotically stable. From the discussion above, we have the following results.

Theorem 2.1 For (1.1), we have:

(i) If $R_{0}<1$, the disease-free equilibrium $E_{1}(A / \mu, 0,0,0)$ is locally asymptotically stable; if $R_{0}>1$, then $E_{1}$ is unstable.

(ii) If $R_{0}>1$, system (1.1) has a unique epidemic equilibrium $E_{2}\left(S^{*}, E^{*}, I^{*}, R^{*}\right)$ which is locally asymptotically stable.

\section{Globally asymptotical stability}

In this section, we will study the global asymptotically stable of $E_{1}(A / \mu, 0,0,0)$ and $E_{2}\left(S^{*}, E^{*}, I^{*}, R^{*}\right)$ by Lyapunov functionals and LaSalle's invariance principle. Noticing that $E(t)$ does not appear in the first, the third, and the fourth equations of (1.1), we only consider the following subsystem of (1.1):

$$
\begin{aligned}
& \frac{d S(t)}{d t}=A-\mu S(t)-\frac{\beta S(t) I(t)}{1+a S(t)+b I(t)}, \\
& \frac{d I(t)}{d t}=\frac{\beta e^{-\mu \tau} S(t-\tau) I(t-\tau)}{1+a S(t-\tau)+b I(t-\tau)}+\delta R(t)-(\mu+\gamma+\epsilon) I(t), \\
& \frac{d R(t)}{d t}=\gamma I(t)-(\mu+\delta) R(t) .
\end{aligned}
$$

Obviously, system (3.1) always has an equilibrium $E_{1}^{0}(A / \mu, 0,0)$. Also, when $R_{0}>1$, system (3.1) has a unique positive equilibrium $E_{2}^{0}\left(S^{*}, I^{*}, R^{*}\right)$, where $S^{*}, I^{*}, R^{*}$ are defined in (2.1). From the discussion above, it is easy to prove that $E_{1}^{0}(A / \mu, 0,0)$ is locally asymptotically stable when $R_{0}<1$. In addition, an equilibrium $E_{2}^{0}\left(S^{*}, I^{*}, R^{*}\right)$ exists when $R_{0}>1$, which is locally asymptotically stable when $R_{0}>1$. First, we will prove the global asymptotical stability of $E_{1}^{0}(A / \mu, 0,0)$.

Theorem 3.1 If $R_{0}<1$, equilibrium $E_{1}^{0}\left(\frac{A}{\mu}, 0,0\right)$ of system (3.1) is globally asymptotically stable in $R_{+}^{3}$.

Proof Denote $S_{0}=A / \mu$, and $(S(t), I(t), R(t))$ is a solution of system (3.1) with initial condition (1.2). Define

$$
V_{1}(t)=\frac{S_{0}}{1+a S_{0}}\left(\frac{S(t)}{S_{0}}-1-\ln \frac{S(t)}{S_{0}}\right)+e^{\mu \tau} I(t)+\frac{\delta e^{\mu \tau}}{\mu+\delta} R(t),
$$

then

$$
\begin{aligned}
\frac{d V_{1}(t)}{d t}= & \frac{1}{1+a S_{0}}\left(1-\frac{S_{0}}{S(t)}\right)\left(A-\mu S(t)-\frac{\beta S(t) I(t)}{1+a S(t)+b I(t)}\right) \\
& +\frac{\beta S(t-\tau) I(t-\tau)}{1+a S(t-\tau)+b I(t-\tau)}-(\mu+\gamma+\epsilon) e^{\mu \tau} I(t) \\
& +\frac{\delta \gamma e^{\mu \tau}}{\mu+\delta} I(t) .
\end{aligned}
$$


Since $A=\mu S_{0}$, we have

$$
\begin{aligned}
\frac{d V_{1}(t)}{d t}= & -\frac{\mu\left(S(t)-S_{0}\right)^{2}}{S(t)\left(1+a S_{0}\right)}-\frac{\beta I(t)\left(S(t)-S_{0}\right)}{(1+a S(t)+b I(t))\left(1+a S_{0}\right)} \\
& +\frac{\beta S(t-\tau) I(t-\tau)}{1+a S(t-\tau)+b I(t-\tau)}-e^{\mu \tau} \alpha I(t),
\end{aligned}
$$

where $\alpha$ is defined in (2.1). We also can construct the following Lyapunov function:

$$
V(t)=V_{1}(t)+\int_{t-\tau}^{t} \frac{\beta S(\theta) I(\theta)}{1+a S(\theta)} d \theta
$$

From (3.3) and (3.4),

$$
\begin{aligned}
\frac{d V(t)}{d t} & =-\frac{\mu\left(S(t)-S_{0}\right)^{2}}{S(t)\left(1+a S_{0}\right)}+\frac{\beta S_{0}(1+a S(t))}{\left(1+a S_{0}\right)(1+a S(t)+b I(t))} I(t)-e^{\mu \tau} \alpha I(t) \\
& \leq-\frac{\mu\left(S(t)-S_{0}\right)^{2}}{S(t)\left(1+a S_{0}\right)}+\frac{e^{\mu \tau}}{(a A+\mu)(\mu+\delta)}\left(R_{0}-1\right) I(t)
\end{aligned}
$$

When $R_{0}<1$, we know $V^{\prime}(t) \leq 0$ from (3.5). From [10] Theorem 5.3.1, we can define $\mathcal{M}$ to be the maximal invariant subset of $\left\{V^{\prime}(t)=0\right\}$. And $\left\{V^{\prime}(t)=0\right\}$ holds if and only if $S(t)=S_{0}$, $I(t)=0$. From the second equation of (3.1),

$$
0=I^{\prime}(t)=\delta R(t)
$$

i.e. $R(t)=0$. Hence, $V^{\prime}(t)=0$ holds if and only if $(S(t), I(t), R(t))=\left(S_{0}, 0,0\right)$. When $R_{0}<1$, $E_{1}^{0}$ is locally asymptotically stable. From LaSalle's invariance principle for delay differential systems [10], we know $E_{1}^{0}(A / \mu, 0,0)$ is globally asymptotically stable.

Corollary 3.2 If $R_{0}<1, E_{1}(A / \mu, 0,0,0)$ is globally asymptotically stable in $R_{4}^{+}$.

Proof Denote by $(S(t), E(t), I(t), R(t))$ a positive solution of system (1.1) satisfying the initial conditions (1.2) and (1.3). From the second equation of (1.1) and (1.3), we have

$$
E(t)=\int_{t-\tau}^{t} \frac{\beta S(\eta) I(\eta)}{1+a S(\eta)+b I(\eta)} e^{-\eta(t-\eta)} d \eta .
$$

From Theorem 3.1, if $R_{0}<1$,

$$
\lim _{t \rightarrow+\infty} S(t)=\frac{A}{\mu}, \quad \lim _{t \rightarrow+\infty} I(t)=0, \quad \lim _{t \rightarrow+\infty} R(t)=0 .
$$

From (3.6) and (3.7)

$$
\begin{aligned}
\lim _{t \rightarrow+\infty} E(t) & =\lim _{t \rightarrow+\infty} \int_{t-\tau}^{t} \frac{\beta S(\eta) I(\eta)}{1+a S(\eta)+b I(\eta)} e^{-\eta(t-\eta)} d \eta \\
& =\lim _{t \rightarrow+\infty}\left[\frac{\beta S(t) I(t)}{1+a S(t)+b I(t)}-\frac{\beta e^{-\mu \tau} S(t-\tau) I(t-\tau)}{1+a S(t-\tau)+b I(t-\tau)}\right] \\
& =0 .
\end{aligned}
$$


Noting if $R_{0}<1$, the disease-free equilibrium $E_{1}(A / \mu, 0,0,0)$ of system (1.1) is locally asymptotically stable, hence $E_{1}$ is globally asymptotically stable.

Next, we will prove the globally asymptotically stability of equilibrium $E_{2}$ of (1.1). First, the globally asymptotically stability of equilibrium $E_{2}^{0}$ of (3.1) is given.

Theorem 3.3 If $R_{0}>1$, epidemic equilibrium of system (3.1) $E_{2}^{0}\left(S^{*}, I^{*}, R^{*}\right)$ is globally asymptotically stable in $R_{+}^{3}$.

Proof Let $(S(t), I(t), R(t))$ be a positive solution of system (3.1) with initial conditions (1.2), and define

$$
\begin{aligned}
V_{20}(t)= & S(t)-S^{*}-\int_{S^{*}}^{S(t)} \frac{1+a \eta+b I^{*}}{1+a S^{*}+b I^{*}} \frac{S^{*}}{\eta} d \eta+e^{\mu \tau}\left(I(t)-I^{*}-I^{*} \ln \frac{I(t)}{I^{*}}\right) \\
& +\frac{\delta e^{\mu \tau}}{\mu+\delta}\left(R(t)-R^{*}-R^{*} \ln \frac{R(t)}{R^{*}}\right),
\end{aligned}
$$

then

$$
\begin{aligned}
\frac{d V_{20}}{d t}= & \left(1-\frac{1+a S(t)+b I^{*}}{1+a S^{*}+b I^{*}} \frac{S^{*}}{S(t)}\right)\left(A-\mu S(t)-\frac{\beta S(t) I(t)}{1+a S(t)+b I(t)}\right) \\
& +\left(1-\frac{I^{*}}{I(t)}\right)\left[\frac{\beta S(t-\tau) I(t-\tau)}{1+a S(t-\tau)+b I(t-\tau)}+\delta e^{\mu \tau} R(t)-(\mu+\gamma+\epsilon) e^{\mu \tau} I(t)\right] \\
& +\frac{\delta e^{\mu \tau}}{\mu+\delta}\left(1-\frac{R^{*}}{R(t)}\right)[\gamma I(t)-(\mu+\delta) R(t)] \\
= & \left(1-\frac{1+a S(t)+b I^{*}}{1+a S^{*}+b I^{*}} \frac{S^{*}}{S(t)}\right)\left[\mu\left(S^{*}-S(t)\right)+\frac{\beta S^{*} I^{*}}{1+a S^{*}+b I^{*}}-\frac{\beta S(t) I(t)}{1+a S(t)+b I(t)}\right] \\
& +\left(1-\frac{I^{*}}{I(t)}\right)\left[\frac{\beta S(t-\tau) I(t-\tau)}{1+a S(t-\tau)+b I(t-\tau)}-(\mu+\gamma+\epsilon) e^{\mu \tau} I(t)\right]+\delta e^{\mu \tau} R(t) \\
& +\frac{\delta e^{\mu \tau}}{\mu+\delta}\left[\gamma I(t)-(\mu+\delta) R(t)-\gamma I(t) \frac{R^{*}}{R(t)}+(\mu+\delta) R^{*}\right] .
\end{aligned}
$$

Define

$$
\begin{aligned}
V_{21}(t)= & \beta \int_{t-\tau}^{t} \frac{S(\eta) I(\eta)}{1+a S(\eta)+b I(\eta)} \\
& -\frac{S^{*} I^{*}}{1+a S^{*}+b I^{*}}-\frac{S^{*} I^{*}}{1+a S^{*}+b I^{*}} \ln \frac{\left(1+a S^{*}+b I^{*}\right) S(\eta) I(\eta)}{S^{*} I^{*}[1+a S(\eta)+b I(\eta)]} d \eta .
\end{aligned}
$$

Construct the following Lyapunov function:

$$
V(t)=V_{20}(t)+V_{21}(t),
$$

then

$$
\begin{aligned}
\frac{d V_{2}(t)}{d t}= & \left(1-\frac{1+a S(t)+b I^{*}}{1+a S^{*}+b I^{*}} \frac{S^{*}}{S(t)}\right)\left[\mu\left(S^{*}-S(t)\right)+\frac{\beta S^{*} I^{*}}{1+a S^{*}+b I^{*}}\right] \\
& +\frac{1+a S(t)+b I^{*}}{1+a S^{*}+b I^{*}} \frac{S^{*}}{S(t)} \frac{\beta S(t) I(t)}{1+a S+b I(t)}-(\mu+\gamma+\epsilon) e^{\mu \tau} I(t)-\delta e^{\mu \tau} R(t) \frac{I^{*}}{I(t)}
\end{aligned}
$$




$$
\begin{aligned}
& -\frac{I^{*}}{I(t)} \frac{\beta S(t-\tau) I(t-\tau)}{1+a S(t-\tau)+b I(t-\tau)}+(\mu+\gamma+\epsilon) e^{\mu \tau} I^{*}+\frac{\delta e^{\mu \tau}}{\mu+\delta} \gamma I(t) \\
& -\frac{\delta e^{\mu \tau}}{\mu+\delta} \gamma I(t) \frac{R^{*}}{R(t)}+\delta e^{\mu \tau} R^{*} \\
& -\frac{\beta S^{*} I^{*}}{1+a S^{*}+b I^{*}} \ln \frac{1+a S^{*}+b I^{*}}{S^{*} I^{*}} \frac{S(t) I(t)}{1+a S(t)+b I(t)} \\
& +\frac{\beta S^{*} I^{*}}{1+a S^{*}+b I^{*}} \ln \frac{1+a S^{*}+b I^{*}}{S^{*} I^{*}} \frac{S(t-\tau) I(t-\tau)}{1+a S(t-\tau)+b I(t-\tau)} \\
& =\left(1-\frac{1+a S(t)+b I^{*}}{1+a S^{*}+b I^{*}} \frac{S^{*}}{S(t)}\right)\left[\mu\left(S^{*}-S(t)\right)+\frac{\beta S^{*} I^{*}}{1+a S^{*}+b I^{*}}\right] \\
& +\frac{1+a S(t)+b I^{*}}{1+a S^{*}+b I^{*}} \frac{S^{*}}{S(t)} \frac{\beta S(t) I(t)}{1+a S(t)+b I(t)}-(\mu+\gamma+\epsilon) e^{\mu \tau} I(t) \\
& -\frac{I^{*}}{I(t)} \frac{\beta S(t-\tau) I(t-\tau)}{1+a S(t-\tau)+b I(t-\tau)}-\delta e^{\mu \tau} R(t) \frac{I^{*}}{I(t)}+(\mu+\gamma+\epsilon) e^{\mu \tau} I^{*} \\
& +\frac{\delta e^{\mu \tau}}{\mu+\delta} \gamma I(t)-\frac{\delta e^{\mu \tau}}{\mu+\delta} \gamma I(t) \frac{R^{*}}{R(t)}+\delta e^{\mu \tau} R^{*} \\
& -\frac{\beta S^{*} I^{*}}{1+a S^{*}+b I^{*}} \ln \frac{1+a S^{*}+b I^{*}}{S^{*} I^{*}} \frac{S(t) I(t)}{1+a S(t)+b I(t)} \\
& +\frac{\beta S^{*} I^{*}}{1+a S^{*}} \ln \frac{1+a S^{*}}{S^{*} I^{*}} \frac{S(t-\tau) I(t-\tau)}{1+a S(t-\tau)+b I(t-\tau)} \\
& =\left(1-\frac{1+a S(t)+b I^{*}}{1+a S^{*}+b I^{*}} \frac{S^{*}}{S(t)}\right)\left[\mu\left(S^{*}-S(t)\right)+\frac{\beta S^{*} I^{*}}{1+a S^{*}+b I^{*}}\right] \\
& +\frac{1+a S(t)+b I^{*}}{1+a S^{*}+b I^{*}} \frac{S^{*}}{S(t)} \frac{\beta S(t) I(t)}{1+a S(t)+b I(t)} \\
& -(\mu+\gamma+\epsilon) e^{\mu \tau} I^{*} \frac{I(t)}{I^{*}}-\frac{\beta S^{*} I^{*}}{1+a S^{*}+b I^{*}} \frac{\left(1+a S^{*}+b I^{*}\right) S(t-\tau) I(t-\tau)}{S^{*} I(t)[1+a S(t-\tau)+b I(t-\tau)]} \\
& -\delta e^{\mu \tau} R^{*} \frac{I^{*}}{R^{*}} \frac{R(t)}{I(t)}+e^{\mu \tau}(\mu+\gamma+\epsilon) I^{*}+\frac{\delta e^{\mu \tau}}{\mu+\delta} \gamma I^{*} \frac{I(t)}{I^{*}}-\frac{\gamma \delta}{\mu+\delta} e^{\mu \tau} I^{*} \frac{R^{*}}{I^{*}} \frac{I(t)}{R(t)} \\
& +\frac{\delta e^{\mu \tau}}{\mu+\delta} \gamma I^{*}+\frac{\beta S^{*} I^{*}}{1+a S^{*}+b I^{*}} \ln \frac{S(t-\tau) I(t-\tau)[1+a S(t)+b I(t)]}{S(t) I(t)[1+a S(t-\tau)+b I(t-\tau)]} \\
& =\left(1-\frac{1+a S(t)+b I^{*}}{1+a S^{*}+b I^{*}} \frac{S^{*}}{S(t)}\right)\left[\mu\left(S^{*}-S(t)\right)+\frac{\beta S^{*} I^{*}}{1+a S^{*}+b I^{*}}\right] \\
& +\frac{1+a S(t)+b I^{*}}{1+a S^{*}+b I^{*}} \frac{S^{*}}{S(t)} \frac{\beta S(t) I(t)}{1+a S(t)+b I(t)}-\frac{\beta S^{*} I^{*}}{1+a S^{*}+b I^{*}} \frac{I(t)}{I^{*}} \\
& -\frac{\beta S^{*} I^{*}}{1+a S^{*}+b I^{*}} \frac{1+a S^{*}+b I^{*}}{S^{*} I(t)} \frac{S(t-\tau) I(t-\tau)}{1+a S(t-\tau)+b I(t-\tau)}+\frac{\beta S^{*} I^{*}}{1+a S^{*}} \\
& +\frac{\beta S^{*} I^{*}}{1+a S^{*}} \ln \frac{S(t-\tau) I(t-\tau)[1+a S(t)+b I(t)]}{S(t) I(t)[1+a S(t-\tau)+b I(t-\tau)]} \\
& +\frac{\gamma \delta}{\mu+\delta} e^{\mu \tau} I^{*}\left(2-\frac{I^{*}}{R^{*}} \frac{R(t)}{I(t)}-\frac{R^{*}}{I^{*}} \frac{I(t)}{R(t)}\right) \\
& =\mu \frac{\left(S(t)-S^{*}\right)^{2}\left(1+b I^{*}\right)}{S(t)\left(1+a S^{*}+b I^{*}\right)}+\frac{\gamma \delta}{\mu+\delta} e^{\mu \tau} I^{*}\left(2-\frac{I^{*}}{R^{*}} \frac{R(t)}{I(t)}-\frac{R^{*}}{I^{*}} \frac{I(t)}{R(t)}\right) \\
& +\frac{\beta S^{*} I^{*}}{1+a S^{*}+b I^{*}}\left[2-\frac{S^{*}}{S(t)} \frac{1+a S(t)+b I^{*}}{1+a S^{*}+b I^{*}}+\frac{I(t)}{I^{*}} \frac{1+a S(t)+b I^{*}}{1+a S(t)+b I(t)}-\frac{I(t)}{I^{*}}\right.
\end{aligned}
$$




$$
\begin{aligned}
& -\frac{1+a S^{*}+b I^{*}}{S^{*} I(t)} \frac{S(t-\tau) I(t-\tau)}{1+a S(t-\tau)+b I(t-\tau)} \\
& \left.+\ln \frac{S(t-\tau) I(t-\tau)[1+a S(t)+b I(t)]}{S(t) I(t)[1+a S(t-\tau)+b I(t-\tau)]}\right] \\
= & \mu \frac{\left(S(t)-S^{*}\right)^{2}\left(1+b I^{*}\right)}{S(t)\left(1+a S^{*}+b I^{*}\right)}+\frac{\gamma \delta}{\mu+\delta} e^{\mu \tau} I^{*}\left(2-\frac{I^{*}}{R^{*}} \frac{R(t)}{I(t)}-\frac{R^{*}}{I^{*}} \frac{I(t)}{R(t)}\right) \\
& +\frac{\beta S^{*} I^{*}}{1+a S^{*}+b I^{*}}\left[1-\frac{S^{*}}{S(t)} \frac{1+a S(t)+b I^{*}}{1+a S^{*}+b I^{*}}+\ln \frac{S^{*}}{S(t)} \frac{1+a S(t)+b I^{*}}{1+a S^{*}+b I^{*}}\right. \\
& +1-\frac{1+a S^{*}+b I^{*}}{S^{*} I(t)} \frac{S(t-\tau) I(t-\tau)}{1+a S(t-\tau)+b I(t-\tau)} \\
& +\ln \frac{1+a S^{*}+b I^{*}}{S^{*} I(t)} \frac{S(t-\tau) I(t-\tau)}{1+a S(t-\tau)+b I(t-\tau)} \\
& \left.+1-\frac{1+a S(t)+b I(t)}{1+a S(t)+b I^{*}}+\ln \frac{1+a S(t)+b I(t)}{1+a S(t)+b I^{*}}\right]+M(t),
\end{aligned}
$$

where

$$
\begin{aligned}
M(t) & =\frac{I(t)}{I^{*}} \frac{1+a S(t)+b I^{*}}{1+a S(t)+b I(t)}-1-\frac{I(t)}{I^{*}}+\frac{1+a S(t)+b I^{*}}{1+a S(t)+b I(t)} \\
& =-\frac{b(1+a S(t))\left(I-I^{*}\right)^{2}}{I^{*}(1+a S(t)+b I(t))\left(1+a S(t)+b I^{*}\right)} \\
& \leq 0 .
\end{aligned}
$$

Here the function

$$
H(t)=1-f(t)+\ln f(t)
$$

is always less than or equal to zero for all $f(t)>0$, and $H(t)=0$ if and only if $f(t)=1$. From the discussion above and the inequality of arithmetic and geometric means, we know $d V_{2}(t) / d t \leq 0, d V_{2}(t) / d t=0$ holds if and only if $S(t)=S^{*}, I(t)=I^{*}, R(t)=R^{*}$. On the other hand, $E_{2}^{0}$ is locally asymptotically stable when $R_{0}>1$. It follows that $E_{2}^{0}$ is globally asymptotically stable from LaSalle's invariance principle and we have a similar proof for Theorem 3.1.

Corollary 3.4 If $R_{0}>1$, the epidemic equilibrium $E_{2}\left(S^{*}, E^{*}, I^{*}, R^{*}\right)$ of system (1.1) is globally asymptotically stable in $R_{+}^{4}$.

\section{Numerical simulations}

To illustrate the theoretical results obtained in this paper, we will give some simulations. The parameter values are chosen to be $A=0.9, \beta=0.3, \mu=0.2, \delta=0.2, \gamma=0.2, a=0.05$, $b=0.6$, and the initial condition $S(0)=0.9, E(0)=5.1, I(0)=4.0, R(0)=3.0$. If we take $\tau=5$, this means $R_{0}=0.810836$. By Corollary 3.4, we know that the disease-free equilibrium $E_{0}$ is globally stable (see Figure $1(\mathrm{a})$ );

Figure 1(b) shows the dynamics of the human population $S_{h}, E_{h}, I_{h}, R_{h}$ in (1.1) when $\tau=0.5$, and other the parameter values remain invariant. Under this condition, we can obtain $R_{0}=1.99434$. Figure $1(\mathrm{~b})$ shows that the trajectories of system (1.1) converge to the malaria-infected equilibrium. 


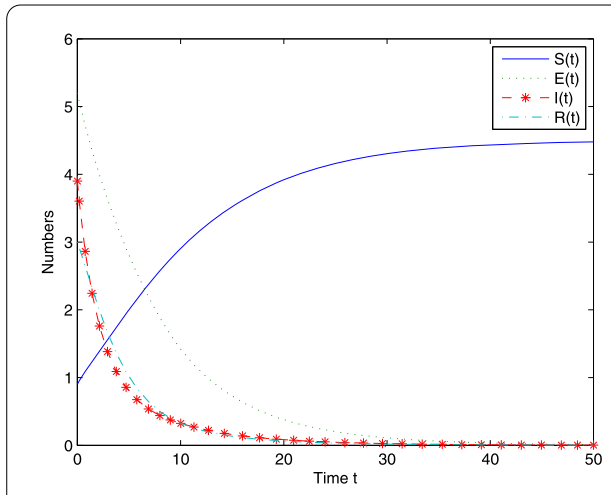

(a) The disease-free equilibrium is globally stable when $R_{0}<1$.

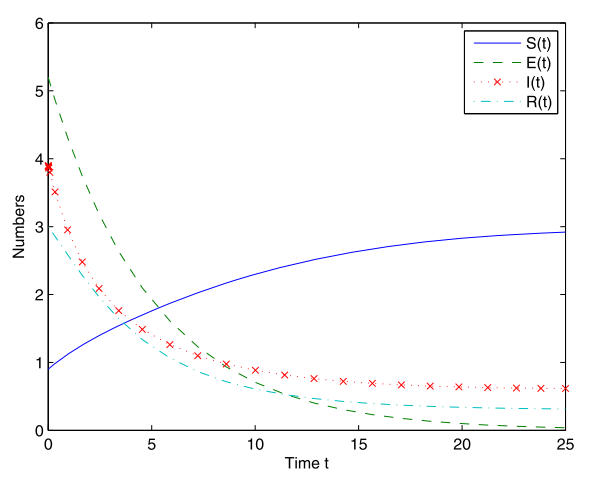

(b) The epidemic equilibrium is globally stable when $R_{0}>1$.

Figure 1 The trajectories of system (1.1).

Figure 2 The relationship between $R_{0}$ and parameter $a$.

In Figure 2, we give the relationship between $R_{0}$ and parameter $a$ and find that the infection force will become lower with increasing of the crowding effect of the susceptible population.

\section{Conclusions and discussion}

In this paper, we have proposed a tuberculosis model with prevention effect and latent delay. We summarize our results of this model in Section 2. The threshold condition $R_{0}$ is given in Section 3. The complete proof of their globally asymptotical stability appears in Section 4. From the expression of the basic reproduction number $R_{0}$ of our model, we know that, in order to control the diseases described in our model, a reasonable strategy should be reduce the threshold condition number $R_{0}$ to below one, and the latent delay has some role to play in controlling this kind of disease, so extending the latent period can reduce the infectiousness of these diseases effectively.

Another purpose of this paper is to study the effect of prevention in our model, the results show the basic reproduction number only depends on parameter 'a' rather than 'b', and this means that the infectiousness of the diseases described in our model is mainly related to the prevention effect of the susceptible rather than infected infectious individual. 
Competing interests

The authors declare that they have no competing interests.

\section{Authors' contributions}

The authors declare that the study was realized in collaboration with the same responsibility. All authors read and approved the final manuscript.

\section{Acknowledgements}

This research has been partially supported by the Ph.D. Fellowship of Xidian University (No. 7214614503) and Chinese Universities Scientific Fund (No. 7215614501). The authors are very grateful to the editor and the anonymous referees for their valuable comments and suggestions, which greatly improved the presentation of this work.

\section{Received: 11 November 2015 Accepted: 8 August 2016 Published online: 30 August 2016}

\section{References}

1. World Health Organization: Global Tuberculosis Report 2012. World Health Organization, Geneva (2012)

2. Huo, HF, Dang, SJ, Li, YN: Stability of a two-strain tuberculosis model with general contact rate. Abstr. Appl. Anal. 2010, 31 (2010)

3. Zhang, JH, Feng, GT: Global stability for a tuberculosis model with isolation and incomplete treatment. Comput. Appl. Math. 34, 1237-1249 (2015)

4. Feng, ZL, Castillo-Chavez, C, Capurro, AF: A model for tuberculosis with exogenous reinfection. Theor. Popul. Biol. 57, 235-247 (2000)

5. Feng, ZL, lannelli, M, Milner, F: A two-strain tuberculosis model with age of infection. SIAM J. Appl. Math. 62, 1634-1656 (2002)

6. Yang, YL, Li, JQ, Zhou, YC: Global stability of two tuberculosis models with treatment and self-cure. Rocky Mt. J. Math. 42, 1367-1386 (2012)

7. Liu, L, Zhang, TL: Global stability for a tuberculosis model. Math. Comput. Model. 54, 836-845 (2011)

8. Li, J, Zhao, YL, Zhu, HP: Bifurcation of an SIS model with nonlinear contact rate. J. Math. Anal. Appl. 432, 1119-1138 (2015)

9. Zhang, XB, Huo, HF, Xiang, H: Dynamics of the deterministic and stochastic SIQS epidemic model with non-linear incidence. Appl. Comput. Math. 243, 546-558 (2014)

10. Hale, JK, Verduyn Lunel, S: Introduction to Functional Differential Equations. Springer, New York (1993)

11. Kuang, Y: Delay Differential Equations with Applications in Population Dynamics. Academic Press, New York (1993)

\section{Submit your manuscript to a SpringerOpen ${ }^{\circ}$ journal and benefit from:}

- Convenient online submission

- Rigorous peer review

- Immediate publication on acceptance

- Open access: articles freely available online

- High visibility within the field

Retaining the copyright to your article 